\title{
The influence of the atrial fibrillation episode duration on the endothelial function in patients treated with pulmonary veins isolation
}

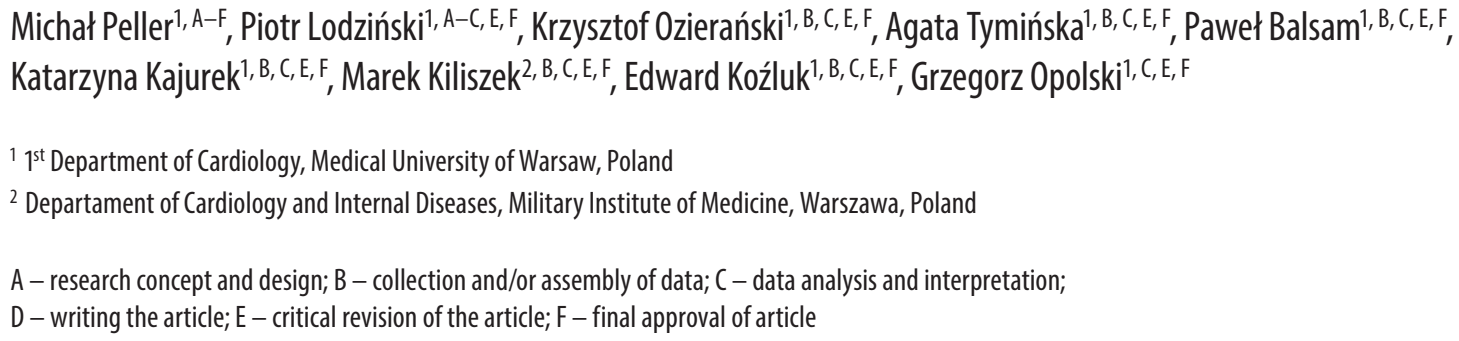

Address for correspondence

Piotr Lodziński

E-mail: piotr.lodzinski@wum.edu.pl

\section{Funding sources}

none declared

\section{Conflict of interest}

none declared

\section{Acknowledgments}

Granted to PM. The grant (number 1WR/NM3/13) was funded by the Medical University of Warsaw.

Received on May 12, 2016

Revised on July 21, 2016

Accepted on November 14, 2016

\begin{abstract}
Background. Atrial fibrillation (AF) is associated with endothelial dysfunctions.

Objectives. The aim of the study was to assess the influence of the duration of an AF episode on the endothelial function.
\end{abstract}

Material and methods. The study included 65 patients with persistent AF qualified for the percutaneous pulmonary veins isolation. Patients were divided into three subgroups with increasing time of the duration of AF episode, as follow: $\leq 7$ months ( $n=24$ patients), from 7 to 14 months ( $n=18$ patients) and $\geq 14$ months ( $n=23$ patients). Concentrations of endothelin-1 (ET-1), thrombomodulin (TM) and VEGF in serum were measured.

Results. Median age in the whole study group was 56 years with $84.6 \%$ of males. Patients with longer lasting AF episode had a higher body mass index and less incidence of heart failure. Median values of ET1, TM and VEGF were 3.1 (2.5-3.5) pg/mL, 3126.0 (2827.2-3594.1) pg/mL and 464.6 (323.6-630.1), respectively. Among increasing tertiles of AF episode duration, median ET-1 serum concentrations were as follows: $3.3(2.8-3.7) \mathrm{pg} / \mathrm{mL}, 3.06(2.6-3.4) \mathrm{pg} / \mathrm{mL}, 2.7(2.3-3.2) \mathrm{pg} / \mathrm{mL}, \mathrm{p}=0.019$, respectively. There was also a trend towards negative association of serum VEGF level with AF episode duration. Serum biomarkers'levels were not associated with total AF duration.

Conclusions. AF episode duration may be associated with the endothelial function, assessed by serum biomarkers. ET-1 serum concentrations are significantly lower in patients with longer AF. ET-1, TM and VEGF have no correlation with total AF duration.

Key words: VEGF, endothelin-1, atrial fibrillation, thrombomodulin, endothelial function

DOI

10.17219/acem/66995

Copyright

Copyright by Author(s)

This is an article distributed under the terms of the

Creative Commons Attribution Non-Commercial License

(http://creativecommons.org/licenses/by-nc-nd/4.0/) 


\section{Introduction}

Endothelium, the innermost layer of the vessels, is responsible for the proper functioning of the cardiovascular system. Its paracrine and endocrine signaling regulates the blood flow, local inflammation response and hemostasis. Endothelial function assessment may be a part of risk stratification in patients with cardiovascular diseases. ${ }^{1}$

Numerous techniques are used for endothelial function evaluation. One of them is the assessment of serum level of endothelium-related biomarkers. Endothelin type 1 (ET-1), protein synthetized and secreted by endothelial cells is a specific and sensitive marker of endothelial dysfunction. ${ }^{2}$ It promotes vasoconstrictions, activates platelets and also influences on myocardial fibrosis. ${ }^{3}$ Endothelial function is also strictly connected with thrombomodulin (TM) serum level, which is elevated during endothelial cells damage. This surface protein has significant anticoagulant properties, which balance procoagulant activities of endothelial dysfunction. ${ }^{4}$ Vascular endothelial grow factor (VEGF) plays a potent role in angiogenesis, endothelial cells proliferation and nitric oxide synthesis by endothelial cells. ${ }^{5}$ Decreased VEGF serum level is the marker of abnormal endothelial function.

Atrial fibrillation (AF) is the most common supraventricular arrhythmia, which occurs in $1.5-2 \%$ of population. ${ }^{6}$ Moreover, AF is one of the cardiovascular diseases in which endothelial dysfunction is well proven. ${ }^{7}$ Previous studies have shown that abnormal endothelial function predisposes the patient to atrial tissue fibrosis, which is a dominant factor responsible for the conversion of paroxysmal to persistent form of the arrhythmia. ${ }^{8}$ However, there is no data on endothelial function changes connected with AF.

The aim of the presented study was to check the potential influence of AF duration on endothelial function, assessed with the serum biomarkers' levels. Influence of total AF duration on endothelial function was additionally assessed.

\section{Material and methods}

\section{Study population}

Endothelial function was prospectively evaluated in a group of 65 consecutive patients with persistent AF, qualified for the percutaneous pulmonary veins isolation between April 2013 and December 2014. According to current guidelines of the European Society of Cardiology, persistent AF was defined as AF episode lasting longer than 7 days or terminated by cardioversion. ${ }^{9}$ Inclusion of the patients was limited to the cases with AF episode lasting longer than 7 days. Qualification for invasive treatment of AF was based on the guidelines of the European
Society of Cardiology. ${ }^{9}$ Exclusion criteria included: neoplastic disease and persistent inflammatory state.

The study was approved by Ethical Review Board (35/2013).

\section{Clinical assessment during hospitalization}

All data according to clinical state, medical history and concomitant diseases was obtained during the hospitalization during which percutaneous pulmonary veins isolation was performed. The baseline laboratory tests included: blood cell morphology, ion levels, liver enzymes activities, creatinine concentration and coagulation tests. The baseline characteristics of patients are presented in Table 1.

Table 1. Baseline characteristics of the study group

\begin{tabular}{|lc|} 
& Study group $(\mathrm{n}=65)$ \\
Age (years) & $56(49-58)$ \\
$\mathrm{BMI}, \mathrm{kg} / \mathrm{m}^{2}$ & $55(84.6 \%)$ \\
White blood cells, $1000 / \mathrm{mcL}$ & $30.1(26.9-32.7)$ \\
Creatinine, $\mathrm{mg} / \mathrm{dL}$ & $7.09(6.43-9.02)$ \\
History of PVI & $1.02(0.91-1.13)$ \\
Coronary disease & $17(26.2 \%)$ \\
Hypertension & $6(9.2 \%)$ \\
Dyslipidemia & $39(60.0 \%)$ \\
History of stroke & $22(33.8 \%)$ \\
Diabetes & $3(4.6 \%)$ \\
Heart failure & $8(12.3 \%)$ \\
Lone AF & $3(4.6 \%)$ \\
History of thyroid disease & $9(13.8 \%)$ \\
\hline
\end{tabular}

$\mathrm{AF}$ - atrial fibrillation; $\mathrm{BMI}$ - body mass index; $\mathrm{PVI}$ - pulmonary veins isolation; values are median (interquartile range) or $\mathrm{n}(\%)$

\section{Duration of atrial fibrillation episode and total atrial fibrillation duration}

Data concerning the duration of atrial fibrillation episode and total atrial fibrillation was based on patients' interviews and medical documentation. To analyze the trends for the concentrations of endothelial function biomarkers, patients were divided into three groups consistent with the duration of AF episode tertiles and the total duration of AF tertiles. In the case of a lack of data about the duration of last AF episode or total AF, patients were excluded from the particular analysis.

\section{Measurements of the biomarkers' levels}

Venous blood samples $(5 \mathrm{~mL})$ were collected at the beginning of the ablation procedure from the vascular sheet placed in the femoral vein. After clotting, all samples were centrifuged for $15 \mathrm{~min}$ at $1000 \times \mathrm{g}$. Serum was removed from the sample directly after centrifugation and stored at the temper- 
ature of $\leq-20^{\circ} \mathrm{C}$ until the end of patients' enrollment. When recruitment of the patients was finished, all serum samples were defrosted and particular assays were performed. Concentrations of ET-1, TM and VEGF were measured using the QuantiGlo Human Endothelin-1 Chemiluminescent Immunoassay (R \& D Systems Inc., USA), the Quantikine Human Thrombomodulin Immunoassay (R \& D Systems Inc., USA) and the QuantiGlo Human VEGF Chemiluminescent Immunoassay (R \& D Systems Inc., USA), respectively. All obtained values were sufficient for the assay ranges.

\section{Statistical analysis}

Continuous variables were presented as mean value \pm standard deviation or as a median and interquartile range (IQR), when normally or non-normally distributed, respectively. Normal distribution of variables was assessed using the Shapiro-Wilk test. Categorical data was presented as absolute and relative frequencies. Statistical significance of trends across increasing duration of AF episode and total AF was assessed: for quantitative variables with Jonckheere-Terpstra test and for qualitative variables - with Cochran-Armitage test for trend. A value of $\mathrm{p}<0.05$ was considered significant for all tests. Statistical analysis was performed using SAS $^{\circ}$ software, v. 9.4.

\section{Results}

A total of 65 patients were included in the analysis. The median age was 56 (49-61) years with $84.6 \%$ of males. Median durations of last AF episode and total AF were 10 (6-19) ranged from 1 to 72 years and 49 (31-72) ranged from 3 to 240 years, respectively. Median values of ET-1, TM and VEGF were 3.1 (2.5-3.5) pg/mL, 3126.0 (2827.2$3594.1) \mathrm{pg} / \mathrm{mL}$ and 464.6 (323.6-630.1), respectively.

\section{Comparison of the groups based on the duration of atrial fibrillation episode}

The analyzed group was divided into 3 subgroups by increasing time of the duration od AF episode. To obtain closest to equal number of patients in each subgroup we used time ranges as follows: $\leq 7$ months ( $\mathrm{n}=24$ patients), from 7 to 14 months ( $\mathrm{n}=18$ patients) and $\geq 14$ months ( $\mathrm{n}=23$ patients). In patients with longer lasting AF episodes we observed a higher body mass index (BMI), less incidence of heart failure and trends towards less frequent usage of calcium channels blockers, angiotensin II receptor blockers, aldosterone antagonists and diuretics. Full comparison of the formed subgroups is presented in

Table 2. Comparison of groups consistent with duration of AF episode tertiles

\begin{tabular}{|c|c|c|c|c|}
\hline & Group $1(n=24)$ & Group $2(n=18)$ & Group $3(n=23)$ & $\mathrm{p}$-value \\
\hline AF episode duration, years & $4(2-6)$ & $11(8-12)$ & $27(18-42)$ & $<0.001$ \\
\hline Age (years) & $55(46-58)$ & $57(53-64)$ & $56(50-58)$ & 0.160 \\
\hline Male & 19 (79.2\%) & $15(83.3 \%)$ & $21(91.3)$ & 0.250 \\
\hline $\mathrm{BMI}, \mathrm{kg} / \mathrm{m}^{2}$ & $32.3(29.2-33.6)$ & $29.1(26.0-32.1)$ & $29.6(26.3-32.1)$ & 0.049 \\
\hline White blood cells, $1000 / \mathrm{mcL}$ & $7.09(6.6-8.9)$ & $7.4(6.5-8.7)$ & $7.1(6.3-9.3)$ & 0.951 \\
\hline Creatinine, mg/dL & $1.03(0.9-1.1)$ & $1.1(1.0-1.2)$ & $1.0(0.8-1.1)$ & 0.252 \\
\hline History of PVI & $8(33.3 \%)$ & $5(27.8 \%)$ & $4(17.4 \%)$ & 0.217 \\
\hline Coronary disease & $2(8.3 \%)$ & $2(11.1 \%)$ & $2(8.7 \%)$ & 0.963 \\
\hline Hypertension & $18(75.0 \%)$ & $9(50.0 \%)$ & $12(52.1 \%)$ & 0.107 \\
\hline Dyslipidemia & $7(29.2 \%)$ & $8(44.4 \%)$ & $7(30.4 \%)$ & 0.916 \\
\hline History of stroke & $1(4.2 \%)$ & $1(5.6 \%)$ & $1(4.4 \%)$ & 0.947 \\
\hline Diabetes & $4(16.7 \%)$ & $2(11.1 \%)$ & $2(8.7 \%)$ & 0.408 \\
\hline Heart failure & $3(12.5 \%)$ & $0(0.0 \%)$ & $0(0.0 \%)$ & 0.042 \\
\hline Lone AF & $3(12.5 \%)$ & $2(11.1 \%)$ & $4(17.4 \%)$ & 0.632 \\
\hline History of thyroid disease & $6(25.0 \%)$ & $2(11.1 \%)$ & $6(26.1 \%)$ & 0.939 \\
\hline \multicolumn{5}{|c|}{ Pharmacotherapy } \\
\hline Beta-adrenolytic & $19(79.2 \%)$ & $15(83.3 \%)$ & $19(82.6 \%)$ & 0.749 \\
\hline Calcium channels blockers & $8(33.3 \%)$ & $3(16.7 \%)$ & $3(13.0 \%)$ & 0.092 \\
\hline ACEl & $15(62.5 \%)$ & $7(38.9 \%)$ & $11(47.8 \%)$ & 0.309 \\
\hline ARB & $4(16.7 \%)$ & $3(16.7 \%)$ & $0(0.0 \%)$ & 0.069 \\
\hline Statins & $7(29.2 \%)$ & $7(38.9 \%)$ & $5(21.7 \%)$ & 0.587 \\
\hline Aldosterone antagonists & $2(8.3 \%)$ & $0(0.0 \%)$ & $0(0.0 \%)$ & 0.099 \\
\hline Diuretics & $6(25.0 \%)$ & $3(16.7 \%)$ & $1(4.4 \%)$ & 0.052 \\
\hline
\end{tabular}

ACEI - angiotensin-converting-enzyme inhibitors; AF - atrial fibrillation; ARB - angiotensin II receptor blockers; BMI - body mass index; PVI - pulmonary veins isolation; atrial fibrillation episode duration - group 1: 1 months; group 2: 7-14 months; Group $3 \geq 14$ months. Values are median (interquartile range) or n (\%). 

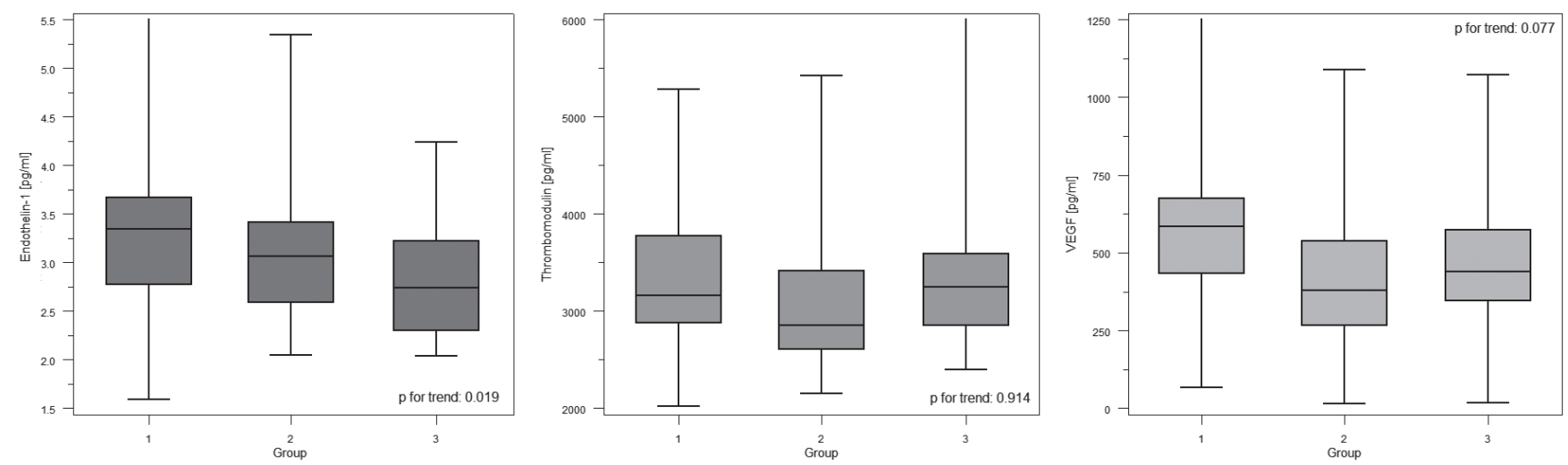

Fig. 1. Concentrations of biomarkers in subgroups divided by duration of atrial fibrillation episode tertiles (VEGF - Vascular Endothelial Growth Factor). Atrial fibrillation episode duration - group 1: 1 months; group 2: 7-14 months; group $3 \geq 14$ months. Boxes present median and interquartile range values.

Table 2. Our analysis showed a significant, negative association of AF episode duration and ET-1 serum concentrations. Among increasing tertiles of AF episode duration, median ET-1 serum concentrations were as follows: $3.3(2.8-3.7) \mathrm{pg} / \mathrm{mL}, 3.1$ (2.6-3.4) pg/mL, 2.7 (2.3-3.2) $\mathrm{pg} / \mathrm{mL}, \mathrm{p}=0.019$, respectively. There was no statistically significant association between AF episode duration and two other biomarkers serum concentration. However, in subgroups we observed a trend towards negative associations of serum VEGF level with AF episode duration: 584.9 (433.0-676.2) pg/mL, $379.2(267.0-537.1) \mathrm{pg} / \mathrm{mL}$ and 439.3 (346.4-573.9) pg/mL, p = 0.077, respectively. Serum levels of ET-1, TM and VEGF are presented in Fig. 1.

\section{Endothelial function in groups based on total atrial fibrillation duration}

Information about total AF duration was obtained in 59 patients. To assess the influence of total AF duration on endothelial function biomarkers concentrations, we divided the analyzed group into three equal subgroups based on AF duration criteria: $\leq 44$ months ( $\mathrm{n}=21$ patients), from 44 to 72 months ( $\mathrm{n}=18$ patients) and $\geq 72$ months ( $n=22$ patients). Comparison of subgroup revealed no statistically significant differences between the subgroup according to clinical characteristics, laboratory test results and pharmacotherapy. There was only a trend towards lower usage of calcium channel blockers in patients with longer lasting AF. We observed no significant differences in ET-1, TM and VEGF serum concentrations between groups. However, patients with longer lasting AF presented lower levels of ET-1 and TM (Table 3).

\section{Discussion}

To the best of our knowledge, this is the first study describing the effects of AF episode duration on endothelial function. The main finding of this study is that the duration of AF episode may influence the endothelial function, assessed by serum concentration of biomarkers. Moreover, we indicated no association between total atrial fibrillation duration and endothelial function.

ET-1, because of its pro-fibrotic properties, may lead to atrial remodeling. Previous studies demonstrated also the influence of endothelial dysfunction on electrical remodeling. ${ }^{9}$ Both of these factors contribute to AF chronicity. ${ }^{10}$ However, there is still not enough data focusing on endothelial dysfunction in chronic form of AF.

The effect of long lasting AF episode on ET-1 serum concentrations presented in the study seems to be unexpected. Because relatively short plasma half-life of ET-1 (about $1.5 \mathrm{~min}$ ), the elevated serum level of this biomarker in the cardiovascular diseases seems to be associated with increased gene expression. ${ }^{11,12}$ Mayyas et al. analyzed the left atrial appendage (LAA) tissue collected during cardiac surgery procedures to show a higher ET-1 concentration in the group of patients with permanent AF then with paroxysmal AF. ${ }^{13}$ However, there was no sig-

Table 3. Comparison of groups consistent with duration of total atrial fibrillation tertiles

\begin{tabular}{|l|c|c|c|} 
& Group 1 $(\mathrm{n}=21)$ & Group 2 $(\mathrm{n}=18)$ & Group $3(\mathrm{n}=20)$ \\
\hline Endothelin-1 $(\mathrm{pg} / \mathrm{mL})$ & $3.2(2.7-3.7)$ & $2.8(2.5-3.5)$ & 0.085 \\
Thrombomodulin $(\mathrm{pg} / \mathrm{mL})$ & $3239(2870-4016)$ & $3074(2827-3444)$ & $3023(2497-3392)$ \\
VEGF $(\mathrm{pg} / \mathrm{mL})$ & $544.3(323.6-579.4)$ & $578.5(416.0-769.9)$ & 0.057 \\
Duration of AF (months) & $24(24-36)$ & $60(48-60)$ & 0.994 \\
\hline
\end{tabular}

AF - atrial fibrillation; VEGF - vascular endothelial growth factor; atrial fibrillation episode duration - group 1: $\leq 44$ months; group 2: $44-72$ months; group $3 \geq 72$ months. Values are median (interquartile range). 
nificant difference between ET-1 tissue concentrations in persistent and paroxysmal AF patients. Partially, higher ET-1 level may be related to the prevalence of concomitant diseases in the group of patients with permanent AF. Previous studies showed no relation between successful cardioversion of persistent AF and short-term reduction of ET-1 concentration. ${ }^{14}$ However, Dezsi et al. presented a rapid decrease of ET-1 level after catheter ablation or modification of atrio-ventricular junction due to supraventricular arrhythmias. ${ }^{15}$ The authors suggest that the heart rhythm is strictly associated with serum ET-1 level. Therefore, a longer lasting episode of AF may result in the adaptation to potential tachyarrhythmia and also in the reduction of ET-1 synthesis.

Mondilo et al. presented that the serum level of TM is significantly higher in patients with chronic, nonrheumatic AF compared with control subjects with sinus rhythm. ${ }^{16}$ By its anticoagulant properties, TM plays an important role in the prevention of thromboembolic events, which are essential a complication of AF. ${ }^{6}$ The lower serum level of TM is associated with endothelial dysfunction and also with an increased procoagulant state. In the presented study we did not reveal a significant correlation between the duration of the AF episode and TM serum level. According to our results, it seems that coagulant properties measured by TM concentration may not be associated with the duration of chronic arrhythmia.

VEGF has vast effects in patients with AF. It promotes an inflammatory response, endothelium proliferation, angiogenesis and also plays a dual role in the coagulant pathway. ${ }^{17,18}$ Scridon A, et al. assessed the VEGF level in particular parts of the vascular system in patients with AF. ${ }^{19}$ Serum concentration of VEGF in the left atrium was significantly higher in patients with paroxysmal AF, compared with control subjects. Interestingly, the VEGF levels in patients with persistent AF and control subjects were similar. Authors colligated higher concentrations of VEGF with pulsatile vascular and atrial stretch. In patients with persistent AF, due to atrial fibrosis, pulsatile stress is decreased. It may results in lower concentrations of VEGF. Results of our study confirmed this theory. We observed a trend toward lower VEGF serum levels in patients with longer lasting AF episode.

The presented study indicated that the duration of AF episode may be associated with endothelial function, assessed by serum biomarkers. ET-1 serum concentrations were significantly lower in patients with longer episodes of AF. We did not find correlations between endothelial function and total AF duration.

\section{Study limitations}

One of the main limitations of the presented study was the precise assessment of AF episodes duration. We tried to obtain this data based on medical documentation. However, this was not possible for every patient.
In these situations, the medical history, including performed procedures and symptoms of the arrhythmia, was collected.

Authors were also faced with the inability to create three groups with equal number of patients consistent with the duration of the AF episode. This situation was associated with the inclusion of patients with the same duration of AF episodes. However, methods of subgroup formation were planned to obtain the maximal equality of the number of patients inside the groups.

A certain restriction of our research was the small size of the groups. Probably because of the association of serum VEGF level with AF episode, the duration did not reach statistical significance.

Obviously some of concomitant diseases may influence the endothelial function. To reduce this bias, the authors focused on a group of patients with persistent AF treated with pulmonary veins isolation. Patients who qualified for this procedure are relatively young and the incidence of diseases, which can influence endothelial function, is low.

\section{References}

1. Halcox JP, Schenke WH, Zalos G, el al. Prognostic value of coronary vascular endothelial dysfunction. Circulation. 2002;106:653-658.

2. Houde M, Desbiens L, D'Orléans-Juste P. Endothelin-1: Biosynthesis, signaling and vasoreactivity. Adv Pharmacol. 2016;77:143-175.

3. Böhm F, Pernow J. The importance of endothelin-1 for vascular dysfunction in cardiovascular disease. Cardiovasc Res. 2007;76:8-18.

4. Ito T, Kakihana Y, Maruyama I. Thrombomodulin as an intravascular safeguard against inflammatory and thrombotic diseases. Expert Opin Ther Targets. 2016;20:151-158.

5. He H, Venema VJ, Gu X, Venema RC, Marrero MB, Caldwell RB. Vascular endothelial growth factor signals endothelial cell production of nitric oxide and prostacyclin through flk-1/KDR activation of c-Src. J Biol Chem. 1999;274:25130-25135.

6. January CT, Wann LS, Alpert JS, el al. American College of Cardiology/American Heart Association. Task Force on Practice Guidelines. 2014 AHA/ACC/HRS guideline for the management of patients with atrial fibrillation: A report of the American College of Cardiology/ American Heart Association Task Force on Practice Guidelines and the Heart Rhythm Society. J Am Coll Cardiol. 2014;64:e1-76.

7. Krishnamoorthy S, Lim SH, Lip GY. Assessment of endothelial (dys) function in atrial fibrillation. Ann Med. 2009;41:576-590.

8. Iwasaki YK, Nishida K, Kato T, Nattel S. Atrial fibrillation pathophysiology: Implications for management. Circulation. 2011; 24:2264-2274.

9. Yagi S, Akaike M, Aihara K, el al. Endothelial nitric oxide synthaseindependent protective action of statin against angiotensin iiinduced atrial remodeling via reduced oxidant injury. Hypertension. 2010;55:918-923.

10. Wijffels MC, Kirchhof CJ, Dorland R, Allessie MA. Atrial fibrillation begets atrial fibrillation. A study in awake chronically instrumented goats. Circulation. 1995;92:1954-1968.

11. Jiao W, Xu J, Zheng J, Shen Y, Lin L, Li J. Elevation of circulating big endothelin-1: An independent prognostic factor for tumor recurrence and survival in patients with esophageal squamous cell carcinoma. BMC Cancer. 2008;8:334

12. Stow LR, Jacobs ME, Wingo CS, Cain BD. Endothelin-1 gene regulation. FASEB J. 2011;25:16-28.

13. Mayyas F, Niebauer M, Zurick A, el al. Association of left atrial endothelin-1 with atrial rhythm, size, and fibrosis in patients with structural heart disease. Circ Arrhythm Electrophysiol. 2010;3:369-379.

14. Wozakowska-Kapłon B, Bartkowiak R, Janiszewska G, Grabowska U. Does atrial fibrillation affect plasma endothelin level? Cardiol J. 2010;17:471-476. 
15. Dézsi CA, Szücs A, Szücs G, el al. Short-term effect of rate control on plasma endothelin levels of patients with tachyarrhythmias. Exp Biol Med (Maywood). 2006;231:852-856.

16. Mondillo S, Sabatini L, Agricola E, el al. Correlation between left atrial size, prothrombotic state and markers of endothelial dysfunction in patients with lone chronic nonrheumatic atrial fibrillation. Int J Cardiol. 2000;75:227-232.
17. Waltenberger J. VEGF resistance as a molecular basis to explain the angiogenesis paradox in diabetes mellitus. Biochem Soc Trans. 2009;37:1167-1170.

18. Kliche $S$, Waltenberger J. VEGF receptor signaling and endothelial function. IUBMB Life. 2001;52:61-66.

19. Scridon A, Morel E, Nonin-Babary E, Girerd N, Fernandez C, Chevalier $P$. Increased intracardiac vascular endothelial growth factor levels in patients with paroxysmal, but not persistent atrial fibrillation. Europace. 2012;14:948-953. 